\title{
TRH attenuates scopolamine-induced memory impairment in humans
}

\author{
Susan E. Molchan ${ }^{1}$, Alan M. Mellow ${ }^{2}$, Brian A. Lawlor ${ }^{1}$, Herbert J. Weingartner ${ }^{3}$, Robert M. Cohen ${ }^{4}$, \\ Martin R. Cohen ${ }^{5}$, and Trey Sunderland ${ }^{1}$ \\ ${ }^{1}$ Unit on Geriatric Psychopharmacology, Section on Clinical Neuropharmacology, Laboratory of Clinical Science, \\ National Institute of Mental Health, 9000 Rockville Pike, Bethesda, MD 20892, USA \\ ${ }^{2}$ Department of Psychiatry, University of Michigan Medical Center, 1103 East Huron, Ann Arbor, MI 48104, USA \\ ${ }^{3}$ Department of Psychology, George Washington University, 2125 G Street, N.W., Washington, DC 20036, USA \\ ${ }^{4}$ Section on Clinical Brain Imaging, Laboratory of Cerebral Metabolism, National Institute of Mental Health, 9000 Rockville Pike, \\ Bethesda, MD 20892, USA \\ ${ }^{5}$ Ensor Research Laboratory, William S. Hall Psychiatric Institute, University of South Carolina School of Medicine, P.O. Box 202, \\ Columbia, SC 29202, USA
}

\begin{abstract}
The brain tripeptide thyrotropin-releasing hormone (TRH) has been demonstrated to facilitate cholinergic neurotransmission. To test its interaction with the cholinergic system in humans, high-dose TRH $(0.5 \mathrm{mg} / \mathrm{kg})$ or placebo was administered intravenously (IV) to normal controls pretreated with scopolamine $(0.5-0.75 \mathrm{mg}$ IV), a centrally active muscarinic antagonist, which has been used to model aspects of the memory impairment of normal aging and of dementia. Compared to placebo, TRH markedly attenuated scopolamine-induced impairment of some measures of memory, most notably on a selective reminding task. This cognitive study is the first in humans to suggest a neuromodulatory effect of a peptide on the cholinergic system, and suggests a facilitatory role for TRH in human memory processes.
\end{abstract}

Key words: Thyrotropin-releasing hormone (TRH) - Neuropeptide - Scopolamine-Memory

Thyrotropin-releasing hormone (TRH), a tripeptide originally described for its effects on the pituitary-thyroid axis, has been found to be distributed throughout the brain (Metcalf 1982). The highest concentrations of TRH receptors in human brain are in the limbic system, specifically in the amygdala, hippocampus, and temporal cortex (Manaker et al. 1986). In animal studies, TRH has been shown to interact with several neurotransmitters. Of these interactions, the positive neuromodulatory effects of TRH on the cholinergic system are the best delineated (Breese et al. 1975; Yarbrough 1979; Kalivas and Horita 1980; Horita et al. 1986). For this reason, TRH and its synthetic analogs have been proposed to have potential therapeutic value in diseases with deficits in the cholinergic system, such as Alzheimer's disease (Yarbrough 1979; Metcalf 1982).

Scopolamine, a centrally active anticholinergic drug that impairs memory, has been shown in previous pharmacologic studies of normal controls to mimic the cognitive impairment of aging or even dementia (Drachman 1977; Caine et al. 1981; Sunderland et al. 1986). In patients with

Offprint requests to: $\mathrm{S}$. Molchan, National Institute of Mental Health, NIH Clinical Center, Building 10, Room 3D41, 9000 Rockville Pike, Bethesda, MD 20892, USA
Alzheimer's disease, loss of cholinergic markers has in fact been correlated with dementia severity (Perry et al. 1978), and patients with Alzheimer's disease have been shown to be more sensitive to the amnestic effect of scopolamine than age-matched controls (Sunderland et al. 1987).

In the present study, we tested the effects of intravenous (IV) TRH in the human scopolamine model of memory impairment. We hypothesized that TRH would reverse the memory impairment caused by scopolamine through its facilitatory effect on the cholinergic system. To obtain central cognitive effects, we administered a very high dose of TRH because it crosses the blood-brain barrier poorly (Metcalf 1982) and is quickly metabolized, having a plasma half-life of only 4-5 min (Bassiri and Utiger 1973).

\section{Methods}

Subjects

Twelve normal paid volunteers ( 8 females, 4 males; mean age $26.9 \pm 7.9$ years; mean years of education $14.4 \pm 1.2$ ) participated in the study after signing informed consent. Body weight of the subjects ranged from 57 to $96 \mathrm{~kg}$ (mean $=76.5 \pm 12.8 \mathrm{~kg}$ ). Volunteers were screened to exclude those with medical or psychiatric illness; all subjects were drug-free for at least 3 weeks prior to the study.

\section{Cognitive tests}

A baseline cognitive battery, which was administered to all subjects, included the Weschler Memory Scale and several tests of attention, memory, and learning as follows.

Selective reminding task. A list of 12 unrelated words was read and subjects were asked to recall the list; missed words were repeated by the examiner and subjects were again asked to recall them (Buschke 1973). This process was repeated for a total of eight trials. Two scores were obtained from this task, one for free recall and the other for consistency of recall, which is the mean number of words recalled prior to reminding by the examiner for each trial. Scores from this task have previously been used to measure episodic learning and memory and effort-demanding memory processes (Weingartner et al. 1983). Scores were re- 
corded for the full task (all eight trials), and were also broken down into the early (1-4) and late (5-8) trials for both free recall and recall consistency.

Vigilance task. Subjects were read a list of 12 categorically related words, 6 of which were repeated; subjects were instructed to signal the examiner upon hearing a word for the second time. The score was the number of correctly identified twice-presented words; this was used as a measure of vigilance-attention. After a distractor task (counting backward), subjects were asked to recall as many items from the list as possible. This free recall score was one of the measures used to evaluate recent or episodic memory. Next, subjects were read a list of 24 categorically related words, 12 of which were from the previous list and 12 of which were new. Subjects were asked to indicate whether a word was old or new. The number of correctly identified words, scored separately for old and new words, was used as a measure of recognition memory. Of the correctly identified old words, subjects were asked to judge the original frequency of presentation (once or twice); the difference between the mean reported frequency of once- and twicepresented words was used as a measure of automatic episodic memory processes (Weingartner et al. 1984).

Category retrieval. Subjects were given two stimuli words and asked to generate as many associated words as possible within a 90 -s period. Subjects were then given two letters and asked to generate words beginning with each letter, also within a 90 -s period. This test was used to assess knowledge or semantic memory (Weingartner et al. 1984).

Digit span. Random sequences of digits were read to subjects who then immediately attempted to recall the digits. Forward digit span was used as a test of attention. The backward digit span, which is considered a test of episodic memory and attention, was also used.

Object recall. In a test commonly used to assess episodic memory, the names of three common objects were read to subjects, who then attempted to recall them after 5 min.

\section{Experimental design}

Following the baseline cognitive battery, subjects participated in two study days, separated by at least $72 \mathrm{~h}$ (Table 1). On each study day, after an overnight fast, an indwelling IV catheter was inserted; scopolamine hydrobromide $(0.5$ or $0.75 \mathrm{mg})$ was then given to subjects by IV push over $1 \mathrm{~min}$. The higher dose of scopolamine was given to subjects weighing over $82 \mathrm{~kg}(N=5)$. This dose was chosen because findings from a previous study showed more

Table 1. Experimental design

\begin{aligned} & \hline Time $(\mathrm{min})$ Procedure \\ & \hline-15 Behavioral ratings \\ & 0 Scopolamine $(0.5$ or $0.75 \mathrm{mg}) \mathrm{IV} \\ &+30$ Behavioral ratings \\ &+45 TRH $(0.5 \mathrm{mg} / \mathrm{kg})$ or Placebo IV \\ &+60 Behavioral ratings \\ &+90 Cognitive testing \\ &+120 Behavioral ratings \\ & \hline\end{aligned}

variability in drug levels after $\mathrm{mg} / \mathrm{kg}$ dosages than after a set dose (Safer and Allen 1971). However, because our range of subject body weights was wide, we decided to give a higher dose to those with the highest weights to try to obtain a more standardized effect from the scopolamine.

Forty-five minutes after subjects received scopolamine, they received either high-dose TRH or placebo by IV push over $1 \mathrm{~min}$. The TRH and placebo were given in a doubleblind manner and were randomized between the 2 study days. The timing of drug administration and of cognitive testing was based on prior studies in humans showing that the cognitive effects of IV scopolamine peak from 90 to 150 min after administration (Safer and Allen 1971) and that central effects of high-dose TRH occur between 30 and 90 min after IV infusion (Mellow et al. 1989). Furthermore, TRH levels in cerebrospinal fluid have been shown to peak $40 \mathrm{~min}$ after administering TRH $(25 \mathrm{mg})$ subcutaneously, with levels still markedly elevated $120 \mathrm{~min}$ after administration (Mitsumoto et al. 1986).

TRH was purchased from Peninsula Laboratories (Belmont, $\mathrm{CA}$ ) and analyzed for purity using high-performance liquid chromatography (HPLC) by the NIH Pharmaceutical Development Service.

The selective reminding, vigilance, and category retrieval tasks were administered once on each study day by a tester blind to the drug condition. The digit span and object recall tasks were performed along with behavioral ratings by the physician investigator at 4 points on each study day (Table 1). Behavioral and physical effects of the drugs were evaluated with both self-ratings and physician ratings. Self-ratings included two visual analog scales (VAS) and the NIMH self-rating scale and symptom checklist (van Kammen and Murphy 1975). The physician completed the modified Brief Psychiatric Rating Scale (BPRS) (Overall and Gorham 1962) and the Pfeiffer Short Portable Mental Status Questionnaire (SPMSQ) (Pfeiffer 1975).

Blood pressure and heart rate were recorded every $5 \mathrm{~min}$ with an automated vital signs monitor (Critikon Inc., Tampa, FL); oral temperature was also measured at the $-15,0,+45,+60,+90,+120$, and +150 min time points on each study day.

\section{Statistical analysis}

Cognitive data were analyzed by one-way repeated measures analysis of variance (ANOVA), with a priori contrast comparisons carried out between drug conditions. Based on our hypothesis that attenuation of the scopolamine-induced memory impairment would occur and on evidence from prior studies (Horita et al. 1986; Yamazaki et al. 1986; Mellow et al. 1989), we used one-tailed probabilities. Also, because of prior studies describing an analeptic effect of TRH (Breese et al. 1975; Horita et a1. 1986; Mellow et al. 1989), we used one-tailed probabilities in the repeated measures ANOVA of the behavioral data to compare scopolamine + placebo with scopolamine + TRH, so that we could maximize detection of a significant stimulant effect on the day subjects received TRH. Thus, one-tailed probabilities were used in the analysis of cognitive data to insure consistency with the one-tailed analysis used for the behavioral data. Because prior studies (Breese et al. 1975; Horita et al. 1986; Mellow et al. 1989) forced us to consider behavioral activation or increased alertness secondary to TRH as a cause of improved cognitive performance, possible rela- 
tionships between cognitive measures and measures of activation were examined by doing correlations between scores from the selective reminding task and the "drowsiness" and "alertness" items from the self-rating scales. A subset of analog scale behavioral measures (items evaluating levels of alertness, concentration, and drowsiness) were compared between drug conditions using the maximum change from baseline (max delta) for each item. Paired $t$-tests (one-tailed) were used for this comparison.

Physiologic data (systolic and diastolic blood pressure, heart rate, and temperature) were analyzed using repeated measures ANOVA using seven pre-selected time points for each protocol day: $-15,0,+45,+60,+90,+120$, and +150 min (Table 1). Retrospectively, we decided to compare the peak recorded blood pressures and heart rates for each study day using paired $t$-tests; one-tailed tests were used because of prior studies reporting TRH to cause a pressor effect peaking from 2 to $5 \mathrm{~min}$ after administration (Borowski et al. 1984). This peak effect would have occurred at about the +50 min time point in our design (5 min after TRH administration), so the blood pressure and heart rate recorded closest to this point were used to compare the peak pressor response between drug conditions.

\section{Results}

\section{Baseline}

On the Weschler Memory Scale, the mean score of all subjects was $115.8 \pm 14.8$; all scores were within normal limits. See Table 2 for scores on the other tests.

\section{Cognitive tests}

Selective reminding task. Subjects performed poorly on this task after scopolamine. Subjects receiving TRH, as compared to placebo, showed a statistically significant attenua-

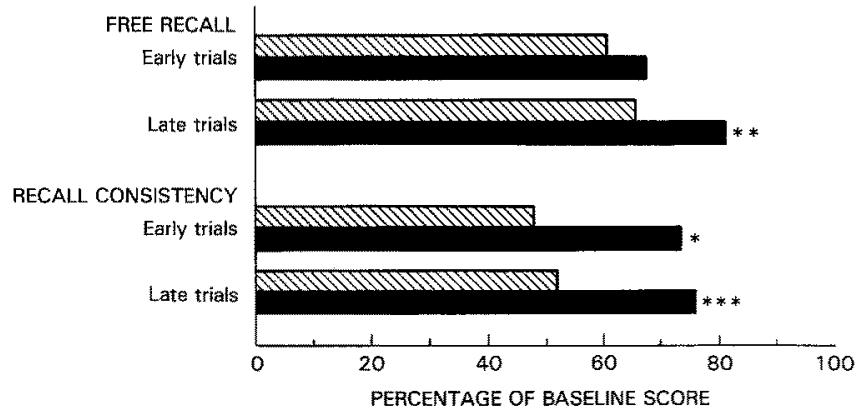

Fig. 1. The graph represents scores from the selective reminding task expressed as percentage of baseline scores. Attenuation of memory impairment after TRH was most evident on the late trials (5-8) of the task for both the free recall and consistency of recall scores. This comparison of late versus early trials represents increased efficiency of learning (Weingartner et al. 1983) $\left(^{*} P<0.05\right.$; ** $P<0.01, * * * P<0.001)$. Scopolamine + placebo; $\square$ Scopolamine + TRH

tion of this scopolamine-induced cognitive impairment. For both free recall and recall consistency scores, most of this improved performance was caused by higher scores on the late trials of the task (Fig. 1).

Vigilance task. The vigilance-attention score reflected no significant drug effect; it was essentially unchanged from baseline after either scopolamine + placebo or scopolamine + TRH. Free recall as assessed by this task declined on the scopolamine + placebo day as compared with baseline, and did not improve significantly on the day subjects received scopolamine + TRH as compared with scopolamine + placebo. On the list of words used to assess recognition memory, a decline from baseline was statistically significant only for the new words (i.e., these words were not as well recognized as words repeated from a prior list). The decline in this measure of recognition memory was attenuated significantly by TRH. Results on the automatic

Table 2. Comparison of the cognitive effects of scopolamine + placebo versus scopolamine + TRH

\begin{tabular}{|c|c|c|c|c|c|}
\hline & Baseline & Scopolamine + placebo & Scopolamine + TRH & $F(1,11)$ & $P$ \\
\hline \multicolumn{6}{|l|}{ Selective reminding } \\
\hline Free recall & $9.34 \pm 1.78$ & $5.89 \pm 1.93$ & $6.98 \pm 1.60$ & 3.72 & $<0.04$ \\
\hline Recall consistency & $0.76 \pm 0.20$ & $0.38 \pm 0.22$ & $0.57 \pm 0.21$ & 10.48 & $<0.004$ \\
\hline \multicolumn{6}{|l|}{ Vigilance task } \\
\hline Vigilance-attention & $5.08 \pm 1.38$ & $5.17 \pm 0.83$ & $5.33 \pm 0.78$ & 0.65 & $N^{a}$ \\
\hline Vigilance free recall & $9.25 \pm 1.42$ & $5.75 \pm 2.42$ & $6.42 \pm 1.62$ & 0.88 & NS \\
\hline Recognition of old words & $10.92 \pm 1.31$ & $10.67 \pm 1.23$ & $10.75 \pm 1.36$ & 0.03 & NS \\
\hline Recognition of new words & $10.17 \pm 1.90$ & $8.92 \pm 2.54$ & $10.33 \pm 1.37$ & 8.05 & $<0.008$ \\
\hline Word frequency & $0.47 \pm 0.33$ & $0.34 \pm 0.40$ & $0.42 \pm 0.32$ & 0.67 & NS \\
\hline \multicolumn{6}{|l|}{ Category retrieval } \\
\hline Words & $22.75 \pm 7.79$ & $16.67 \pm 6.78$ & $16.83 \pm 3.93$ & 0.01 & NS \\
\hline Letters & $19.92 \pm 5.07$ & $14.63 \pm 6.14$ & $17.54 \pm 6.31$ & 26.47 & $<0.001$ \\
\hline \multicolumn{6}{|l|}{ Other cognitive measures } \\
\hline Forward digit span & $7.79 \pm 0.66$ & $7.00 \pm 1.00$ & $7.17 \pm 0.72$ & 1.00 & $N S^{a}$ \\
\hline Backward digit span & $6.04 \pm 0.58$ & $5.36 \pm 0.67$ & $5.83 \pm 0.72$ & 3.38 & $<0.05$ \\
\hline 3 object recall & $2.92 \pm 0.29$ & $1.82 \pm 1.25$ & $2.42 \pm 0.67$ & 2.40 & $<0.08$ \\
\hline
\end{tabular}

${ }^{a}$ Test scores important in showing no significant difference in attention between study conditions

The data are presented as the means $\pm \mathrm{SD} ; \mathrm{NS}=$ not significant 
Table 3. Comparison of the behavioral effects of scopolamine + placebo versus scopolamine + TRH

\begin{tabular}{|c|c|c|c|c|c|c|c|c|}
\hline & \multicolumn{2}{|l|}{ Baseline $^{a}$} & \multicolumn{2}{|l|}{$+60 \min ^{\mathrm{a}}$} & \multicolumn{2}{|l|}{$+120 \min ^{\mathrm{a}}$} & \multirow[t]{2}{*}{$F$} & \multirow[t]{2}{*}{$P$} \\
\hline & $\mathrm{Scop}+\mathrm{Plac}$ & Scop + TRH & Scop + Plac & Scop + TRH & $\mathrm{Scop}+\mathrm{Plac}$ & Scop + TRH & & \\
\hline Restlessness & $8.5 \pm 8.2$ & $8.5 \pm 6.7$ & $16.2 \pm 18.9$ & $20.7 \pm 23.3$ & $13.8 \pm 14.6$ & $15.6 \pm 15.4$ & 1.80 & $<0.12$ \\
\hline Anxious/nervous & $12.9 \pm 14.6$ & $14.0 \pm 12.8$ & $14.3 \pm 14.2$ & $10.7 \pm 8.0$ & $9.9 \pm 8.7$ & $9.3 \pm 7.2$ & 0.03 & $<0.44$ \\
\hline Alert & $58.9 \pm 20.7$ & $70.8 \pm 18.0$ & $29.0 \pm 24.3$ & $37.3 \pm 29.7$ & $31.5 \pm 19.7$ & $40.8 \pm 28.5$ & 2.36 & $<0.80$ \\
\hline $\mathrm{Sad} /$ depressed & $4.1 \pm 2.1$ & $4.7 \pm 1.8$ & $4.2 \pm 2.7$ & $5.5 \pm 3.2$ & $4.7 \pm 2.4$ & $3.8 \pm 1.9$ & 0.50 & $<0.25$ \\
\hline Energetic & $38.9 \pm 24.9$ & $40.6 \pm 23.6$ & $8.4 \pm 7.9$ & $14.5 \pm 14.7$ & $13.1 \pm 10.9$ & $14.8 \pm 15.6$ & 0.15 & $<0.35$ \\
\hline Trouble concentrating & $7.6 \pm 9.6$ & $7.4 \pm 7.5$ & $32.9 \pm 20.3$ & $33.6 \pm 22.4$ & $32.1 \pm 24.2$ & $34.5 \pm 21.7$ & 0.16 & $<0.35$ \\
\hline Fatigued/tired & $17.0 \pm 21.6$ & $17.5 \pm 17.6$ & $39.1 \pm 21.5$ & $29.7 \pm 20.8$ & $33.3 \pm 24.0$ & $30.7 \pm 19.7$ & 0.98 & $<0.18$ \\
\hline Drowsy & $15.2 \pm 18.6$ & $15.7 \pm 18.7$ & $45.8 \pm 24.3$ & $35.6 \pm 26.7$ & $40.9 \pm 23.4$ & $32.2 \pm 24.1$ & 4.31 & $<0.03$ \\
\hline Disoriented & $6.0 \pm 2.7$ & $5.0 \pm 3.0$ & $16.7 \pm 16.8$ & $19.7 \pm 17.7$ & $11.0 \pm 9.5$ & $12.2 \pm 12.9$ & 0.62 & $<0.23$ \\
\hline Disjointed speech & $6.0 \pm 2.7$ & $5.1 \pm 3.2$ & $15.4 \pm 18.9$ & $16.2 \pm 17.1$ & $12.4 \pm 11.1$ & $11.0 \pm 12.2$ & 0.00 & $<0.50$ \\
\hline Euphoric & $6.3 \pm 3.1$ & $5.2 \pm 2.5$ & $6.5 \pm 3.7$ & $7.8 \pm 5.9$ & $5.6 \pm 2.6$ & $7.5 \pm 7.9$ & 0.06 & $<0.41$ \\
\hline Contrary/irritable & $5.7 \pm 2.6$ & $5.2 \pm 2.5$ & $11.9 \pm 15.9$ & $15.7 \pm 19.5$ & $8.3 \pm 5.4$ & $6.7 \pm 3.9$ & 0.16 & $<0.35$ \\
\hline Dry mouth & $5.6 \pm 3.2$ & $7.5 \pm 5.9$ & $54.6 \pm 20.7$ & $46.8 \pm 22.4$ & $42.3 \pm 27.4$ & $48.3 \pm 24.6$ & 1.62 & $<0.21$ \\
\hline Motor retardation & $4.4 \pm 1.4$ & $4.1 \pm 2.0$ & $16.3 \pm 5.4$ & $11.2 \pm 8.5$ & $10.9 \pm 6.0$ & $10.3 \pm 4.7$ & 0.03 & $<0.44$ \\
\hline
\end{tabular}

Subjects completed $100 \mathrm{~mm}(1=$ none, $100=$ severe $)$ visual analog rating scales on the above items. Data are listed for baseline, the +60 , and the +120 min time points (see Table 1)

a Mean $+\mathrm{SD}$

processing task significantly declined after scopolamine as compared with baseline, with no significant improvement after TRH.

Category retrieval. A decline for baseline scores was significant on both the word and letter portions of this test following scopolamine. When subjects received scopolamine + TRH, they had significant attenuation of this decline in their ability to retrieve words for the letter portion of the test only.

Digit span. On the forward digit span, subjects showed a significant decline from baseline after scopolamine, with no significant difference on days they received scopolamine + placebo as compared with scopolamine+TRH. A statistically significant decline from baseline on the backward digit span was also evident after scopolamine; this decline was significantly attenuated on the days subjects received scopolamine $+\mathrm{TRH}$.

Object recall. Subjects showed a significant decline from baseline on object recall after scopolamine, with attenuation of this decline after TRH, at a trend level of statistical significance.

\section{Behavioral measures}

The self-ratings comparing scopolamine + placebo and scopolamine + TRH showed an increase only in the VAS item "drowsy" $[F(1,9)=4.31 ; P<0.04]$ after scopolamine + placebo (Table 3) and in the physical symptom of "chilled/ shivering" (a common, transient side effect of high-dose TRH (Mellow et al. 1989) $[F(1,9)=7.5 ; P<0.01]$ after scopolamine + TRH. No statistically significant correlation was found between the "drowsy" and "alert" items and scores on the selective reminding task. The additional comparison between drug conditions of analog scale measures of alertness, concentration, and drowsiness using the maximum change from baseline (max delta) did not differ significantly on the days subjects received scopolamine + placebo as compared with the days they received scopolamine+
TRH, except on the item "restlessness" which was higher on the scopolamine + TRH day $(t=2.0 ; P<0.04)$. On the physician-rated BPRS and SPMSQ, no significant differences were found between subjects on the two drug conditions.

\section{Physiological measures}

The ANOVA comparing blood pressure, heart rate, and temperature showed no significant differences between scopolamine + placebo and scopolamine + TRH conditions for the time points in Table 1. However, the analysis of the retrospectively chosen peak systolic blood pressure (recorded on average $8 \pm 3.7 \mathrm{~min}$ after TRH or placebo administration) did show a significant difference between the two drug conditions $(t=-2.3 ; 10 \mathrm{df} ; P<0.02)$; after scopolamine + placebo, the mean peak systolic blood pressure was $117.8 \pm 11.7 \mathrm{mmHg}$; and, after scopolamine + TRH, it was $129.5 \pm 16.6 \mathrm{mmHg}$. The same was true for mean peak diastolic blood pressure $(t=-3.6 ; 10 \mathrm{df} ; P<0.003)$, with the mean peaks at $67.9 \pm 11.9 \mathrm{mmHg}$ and $87.3 \pm 16.5 \mathrm{mmHg}$, respectively. The mean peak heart rates were not significantly different between drug conditions.

\section{Discussion}

This study is the first in humans to assess peptidergic neuromodulation of a drug-induced cognitive effect mediated by a classical neurotransmitter system. In addition to scopolamine's pharmacological specificity, involvement of the cholinergic system in attenuated memory impairment is likely because the selective reminding task has been shown to be among the most sensitive of cognitive tests to the effects of scopolamine (Caine et al. 1981; Sunderland et al. 1987). In our study, this task was also the most sensitive to the effects of TRH. The selective reminding task is different from the other tests used because it requires more sustained concentration and cognitive effort. Thus, our data indicate that the cognitive components of information processing most sensitive to TRH are the effort-demanding rather than the more automatic or incidental encoding pro- 
cesses (Hasher and Zacks 1979). Only part of the recognition memory portion of the vigilance task and part of the category retrieval task showed significant differences between the scopolamine + placebo and scopolamine + TRH conditions (Table 2). The combination of cognitive tests used in our paradigm was designed to target specific cognitive domains as explained in the Methods section. It is difficult to evaluate any specific cognitive process (such as episodic memory) with any one test. For example, the selective reminding task requires among other things, both memory and set-shifting capacities. However, attenuation of the scopolamine-induced impairment was also evident on some of the other tests of memory, though not as consistently as on the selective reminding task, as discussed above and shown in Table 2 . These results support our contention that the drugs were affecting memory.

TRH has analeptic effects (Breese et al. 1975; Kalivas and Horita 1980), which may explain the behavioral rating of less drowsiness following TRH, and though it cannot be completely determined how much arousal or attentional changes may have contributed to the attenuated scopolamine-induced memory impairment after administration of TRH; no change in attention or concentration was found on the vigilance-attention and forward digit span tests. In addition, no significant difference between drug conditions was demonstrated when several analog scale measures of attention, concentration, and drowsiness were compared using the maximum change from baseline of these items. In fact, a significant increase on the item "restlessness" was found on the day subjects received TRH. An increase in restlessness, if anything, would tend to have a negative effect on cognitive testing. Also, the fact that cognitive measures of attention were essentially the same between the scopolamine + placebo and scopolamine + TRH conditions, and that significant correlations were not found between cognitive test scores and behavioral measures of alertness supports a more specific effect of TRH on memory and learning. Other investigators have found scopolamine (at doses comparable to those in our paradigm) to have little effect on attention (Safer and Allen 1971; Caine et al. 1981), although there is some disagreement on this point (Dunne and Hartley 1985). Attention is notoriously difficult to measure specifically in humans. In addition, a general arousal effect was not shown in blood pressure and heart rate by the time of cognitive testing, though an increase in blood pressure was found shortly after TRH infusion. Statistically significant differences were not found in these physiological parameters between scopolamine + TRH and scopolamine + placebo after this initial increase on the day subjects received TRH.

Additional data supporting a specific effect of TRH on cognition rather than simply activation are that most attempts to reverse scopolamine-induced memory impairment with stimulant drugs such as amphetamine and methylphenidate have been unsuccessful (Drachman 1977; Bartus 1978; Mewaldt and Ghoneim 1979; Yamazaki et al. 1986). Further supporting a specific effect of TRH through alteration of the cholinergic system is the finding that drugs like physostigmine and arecoline, which enhance cholinergic transmission, clearly attenuate or reverse the cognitive impairment caused by scopolamine in animals and humans (Drachman 1977; Bartus 1978; Sitaram et al. 1978).

While our findings are consistent with results showing a modulatory effect of TRH on the cholinergic system, other studies have shown TRH to increase levels of other neuroactive substances such as catecholamines, indoleamines, and vasopressin (Metcalf 1982; Horita et al. 1986), each of which may have positive memory effects of their own. The absence of a general arousal effect on most behavioral and physiological measures is evidence against (though does not necessarily rule out) a significant involvement of catecholamines, as is the absence of a sustained pressor response evidence against involvement of vasopressin. The involvement of thyroid-stimulating hormone (TSH) or thyroid hormones as mediators of the cognitive effects is unlikely for several reasons: TSH crosses the blood-brain barrier poorly; the high dose of TRH used in our paradigm would not cause supraphysiologic levels of TSH because maximum stimulation of TSH occurs in humans after infusion of about $500 \mu \mathrm{g}$ TRH (Snyder and Utiger 1972; Mitsumoto et al. 1986); and thyroid hormones do not begin to increase until $90 \mathrm{~min}$ after TRH infusion (Jackson 1982). Cognitive testing in our paradigm was completed before that time point.

Possible criticisms of the study relate to the blindness of the subjects and of the staff regarding whether subjects received TRH or placebo. Although our paradigm was set up as a double-blind study, high-dose TRH has some specific side effects, most notably a shivering response, so subjects may have suspected when they received it. Therefore, the informed consent signed by subjects was worded so that they did not know the hypothesized direction of change in cognitive test performance expected after receiving TRH. Fortunately, because the side effects of TRH last for only 5-10 min, they had subsided by the time of cognitive testing, so that the tester remained blind as to whether or not TRH was received.

A significant practice effect contributing to the positive cognitive results was ruled out by the randomization of the TRH and placebo administration. In fact, eight subjects received TRH on the 1st study day, and any overall effect of practice (causing improvement on the 2nd study day) would have tended to occur and favor test performance on the day more subjects received placebo.

Our results are consistent with findings from animal studies showing TRH analogs to attenuate memory and learning impairment produced by scopolamine or by lesions of the predominantly cholinergic septo-hippocampal pathway (Yamazaki et al. 1986; Horita et al. 1987). Moreover, brain areas important for memory and learning (the medial septum-diagonal band of Broca and the nucleus basalis of Meynert) are highly sensitive to one cholinergically mediated central effect of TRH, the analeptic action (Kalivas and Horita 1980; Horita et al. 1986, 1987).

The proposed mechanism of action of TRH in our paradigm includes presynaptic and/or postsynaptic interactions with cholinergic neurons. Presynaptically, TRH has been shown to facilitate cholinergic transmission by stimulating the turnover rate of acetylcholine (ACh) and attenuating the pentobarbital-induced reduction of high-affinity choline uptake (Schmidt 1977). Postsynaptically, TRH increases the excitatory effects of $\mathrm{ACh}$ on cerebral cortical neurons (though this action has not been observed by all investigators) (Winokur and Beckman 1978), and directly excites cholinergic septo-hippocampal neurons (Lamour et al. 1985). To date, no direct action of TRH at the cholinergic receptor has been demonstrated.

This study demonstrates the ability of TRH to reverse 
in part the central anticholinergic effects of scopolamine. Although not all aspects of cognitive function were sensitive to $\mathrm{TRH}$, the demonstrated positive effects engender speculation about other possible neuropeptide modulators and their investigation using similar pharmacologic models of cognitive impairment. Investigations into the therapeutic potential of the cholinergic enhancing properties of highdose TRH are ongoing. In a recent study from our group, patients with Alzheimer's disease showed a modest improvement in memory after acute infusion of high-dose TRH as compared with placebo (Mellow et al. 1989). Further studies using TRH as a neuropharmacological probe are necessary to delineate its psychobiological effects and to study the mechanism of peptidergic neuromodulation.

Acknowledgement. The authors wish to acknowledge the NIMH 6-D nursing staff, Lisa Efron, and Karen Thompson for their assistance and support, James L. Hill for help with cognitive testing and statistical analysis, and Lee Hoffman for editorial assistance.

\section{References}

Bartus RT (1978) Evidence for a direct cholinergic involvement in the scopolamine-induced amnesia in monkeys: evidence of concurrent administration of physostigmine and methylphenidate with scopolamine. Pharmacol Biochem Behav 9:833-836

Bassiri RM, Utiger RD (1973) Metabolism and excretion of exogenous thyrotropin releasing hormone in humans. I Clin Invest $52: 1616-1619$

Borowski GD, Garofano CD, Rose LI, Levy RA (1984) Blood pressure response to thyrotropin-releasing hormone in euthyroid subjects. J Clin Endocrinol Metab 58:197-200

Breese GR, Cott JM, Cooper BR, Prange AJ, Lipton MA, Plotnikoff NP (1975) Effects of thyrotropin-releasing hormone (TRH) on the actions of pentobarbital and other centrally acting drugs. J Pharmacol Exp Ther 193:11-22

Buschke H (1973) Selective reminding for analysis of memory and learning. J Verb Learn Verb Behav 12:543-550

Caine ED, Weingartner $\mathrm{H}$, Ludlow DL, Cudahy EA, Wehry S (1981) Qualitative analysis of scopolamine-induced amnesia. Psychopharmacology 74:74-80

Drachman DA (1977) Memory and cognitive function in man: does the cholinergic system have a specific role? Neurology $27: 783-790$

Dunne MP, Hartley LR (1985) The effects of scopolamine upon verbal memory: evidence for an attentional hypothesis. Acta Psychol 58:205-217

Hasher L, Zacks RT (1979) Automatic and effortful processes in memory. J Exp Psychol [Gen] 108:356-388

Horita A, Carino MA, Lai H (1986) Pharmacology of thyrotropinreleasing hormone. Annu Rev Pharmacol Toxicol 26:311-332

Horita A, Carino MA, Lai $H$ (1987) Preliminary studies of the effects of a TRH analog, MK-771, in an animal model of Alzheimer"s disease. Proc West Pharmacol Soc 30:57-58

Jackson IMD (1982) Thyrotropin-releasing hormone. New Engl J Med 306:145-155

Kalivas PW, Horita A (1980) Thyrotropin-releasing hormone: neurogenesis of actions in the pentobarbital narcotized rat. J Pharmacol Exp Ther 212:203-210

van Kammen DP, Murphy DL (1975) Attenuation of the euphor. iant and activating effects of $d$-and $l$-amphetamine by lithium carbonate treatment. Psychopharmacologia 44:215-224

Lamour Y, Dutar P, Jobert A (1985) Effects of TRH, cyclo-(HisPro) and (3-Me-His)TRH on identified septohippocampal netrons in the rat. Brain Res $331: 343-347$
Manaker S, Eichen A, Winokur A, Rhodes CH, Rainbow TC (1986) Autographic localization of thyrotropin releasing hormone receptors in human brain. Neurology 36:641-646

Mellow AM, Sunderland T, Cohen RM, Lawlor BA, Hill JL, Newhouse PA, Cohen MR, Murphy DL (1989) Acute effects of high-dose thyrotropin releasing hormone infusions in Alzheimer's disease. Psychopharmacology 98:403-407

Metcalf G (1982) Regulatory peptides as a source of new drugs: the clinical prospects for analogues of TRH which are resistant to metabolic degradation. Brain Res Rev 4:389 408

Mewaldt SP, Ghoneim MM (1979) The effects and interactions of scopolamine, physostigmine and methamphetamine on human memory. Pharmacol Biochem Behav 10:205-210

Mitsumoto H, Salgado ED, Negroski D, Hanson MR, Salanga VD, Wilber JF, Wilbourn AJ, Breur AC, Leatherman J (1986) Amyotrophic lateral sclerosis: effects of acute intravenous and chronic subcutaneous administration of thyrotropin-releasing hormone in controlled trials. Neurology 36:152-159

Overall JE, Gorham DR (1962) The Brief Psychiatric Rating Scale. Psychol Rep 10:799-812

Perry EK, Tomlinson BE, Blessed G, Bergman K, Bigson PH, Perry RH (1978) Correlation of cholinergic abnormalities with senile plaques and mental test scores in senile dementia. $\mathrm{Br}$ Med J 2:1457-1459

Pfeiffer E (1975) A short portable mental status questionnaire for the assessment of organic brain deficit in elderly patients. J Am Geriatr Soc 23:433-441

Safer DJ, Allen RP (1971) The central effects of scopolamine in man. Biol Psychiatry 3:347-355

Schmidt DE (1977) Effects of thyrotropin releasing hormone (TRH) on pentobarbital-induced decrease in cholinergic neuronal activity. Psychopharmacol Commun 1:469-473

Sitaram N, Weingartner H, Gillin JC (1978) Human serial learning: enhancement with arecholine and choline impairment with scopolamine. Science 201:274-276

Snyder PJ, Utiger RD (1972) Response to thyrotropin releasing hormone (TRH) in normal man. J Clin Endocrinol Metab $34: 380-385$

Sunderland T, Tariot PN, Weingartner H, Murphy DL, Newhouse PA, Mueller EA, Cohen RM (1986) Pharmacologic modelling of Alzheimer's disease. Prog Neuropsychopharmacol Biol Psychiatry 10:599-610

Sunderland T, Tariot PN, Cohen RM, Weingartner H, Muekker EA, Murphy DL (1987) Anticholinergic sensitivity in patients with dementia of the Alzheimer type and age-matched controls. Arch Gen Psychiatry 44:418-426

Weingartner $\mathrm{H}$, Grafman J, Boutelle W, Kaye W, Martin PR (1983) Forms of memory failure. Science 221:380-382

Weingartner H, Burns D, Diebel R, LeWitt PA (1984) Cognitive impairments in Parkinson's disease: distinguishing between effort-demanding and automatic cognitive processes. Psychiatry Res 11:223-235

Winokur A, Beckman AL (1978) Effects of thyrotropin-releasing hormone, norepinephrine and acetylcholine on the activity of neurons in the hypothalamus, septum and cerebral cortex of the rat. Brain Res 150:205-209

Yamazaki N, Nagaoka N, Nagawa Y (1986) Effect of thyrotropinreleasing hormone and its analog DN-1417 on scopolamineinduced impairment of short-term memory in rats. Jpn J Psychopharmacol 6:359-366

Yarbrough GG (1979) On the neuropharmacology of thyrotropin releasing hormone (TRH). Prog Neurobiol 12:291-312

Received April 3, 1989/Final version June 20, 1989 